\title{
Effects of Sowing Time and Growing Density on Agronomic Traits, Grain Yield, and Grain Quality of Waxy Sorghum Cultivar Hongliangfeng 1
}

\author{
Can Wang ${ }^{1}$, Lingbo Zhou ${ }^{1}$, Guobing Zhang ${ }^{1}$, Yan $\mathrm{Xu}^{1}, \mathrm{Xu} \mathrm{Gao}^{1}$, Ne Jiang ${ }^{1}$, Liyi Zhang ${ }^{1} \&$ Mingbo Shao ${ }^{1}$ \\ ${ }^{1}$ Institute of Upland Food Crops, Guizhou Academy of Agricultural Sciences, Guiyang, Guizhou, China \\ Correspondence: Mingbo Shao, Institute of Upland Food Crops, Guizhou Academy of Agricultural Sciences, \\ Guiyang 550006, Guizhou, China. E-mail: 563189433@qq.com
}

Received: February 16, 2019

Accepted: March 19, 2019

Online Published: May 15, 2019

doi:10.5539/jas.v11n6p12

URL: https://doi.org/10.5539/jas.v11n6p12

\begin{abstract}
The aim of this study was to determine the effects of sowing time and growing density on the yield and quality of grain in waxy sorghum (Sorghum bicolor L. Moench). The main plots were two sowing time: early sowing (5 April) and late sowing (20 April), and the subplots were three growing densities: $0.8 \times 10^{5}, 1.1 \times 10^{5}$, and $1.4 \times$ $10^{5}$ plants/ha. Results showed that sowing time and growing density had significant effects on grain yield and grain quality of waxy sorghum cultivar Hongliangfeng 1. Grain yield, plant height, spike length, culm diameter, grain number per spike, grain weight per plant, 1000-grain weight, protein content, starch content, and amylopectin content were reduced by a delay of sowing time, while the tannin content and amylose content were increased by a delay of sowing time. Grain yield, plant height, spike length, culm diameter, grain number per spike, grain weight per plant, 1000-grain weight, protein content, starch content, and amylopectin content increased and then decreased with the increase of growing density, while the tannin content and amylose content decreased and then increased with the increase of growing density. These results hinted that appropriate sowing time and growing density are key cultivation measures to ensure high yield and good quality in waxy sorghum production.
\end{abstract}

Keywords: growing density, sowing time, grain yield, grain quality, sorghum

\section{Introduction}

Sorghum (Sorghum bicolor L. Moench) is the world's fifth-largest food crop after corn, wheat, rice, and barley, which is widely planted in tropical and semi-arid regions of the world (Awika et al., 2003; Borrell et al., 2014). It is also one of the earliest cultivated cereal crops in China (Ding et al., 2015). Sorghum has gradually become a model crop of cereal crops genome research due to its wide adaptability to environment, strong resistance, and relatively small genome (Paterson et al., 2009; Mace et al., 2013). In general, sorghum is divided into three types, sweet sorghum, forage sorghum, and grain sorghum according to its different purposes (Bibi et al., 2012). Waxy sorghum belongs to grain sorghum, which is the main raw material for brewing Moutai flavor liquor and plays a crucial part in the development of China's regional economy (Wang et al., 2017a; Wang et al., 2017b). With the rapid development of the Moutai flavor liquor industry, the demand for waxy sorghum yield is increasing and the demand for waxy sorghum quality is becoming more and more strict (Zhou et al., 2008; Peng, 2011). Thus, how to improve the grain yield and quality of waxy sorghum is the key to the development of the Moutai flavor liquor industry.

In general, sorghum yield and quality are strongly dependent on climate conditions, fertilization, sowing time, growing density, irrigation, and soil types (Townend et al., 1996; Chipanshi et al., 2003; Ekeleme et al., 2011; Han et al., 2011; Jahanzad, 2013). Sowing time and growing density are two important factors for successful implementation of management measures in sorghum production (Almodares \& Mostafafi Darany, 2006; Marsalis et al., 2010). Sowing time is an essential practice of crop management, which could be manipulated to better control sorghum growth (Ekeleme et al., 2011). Early sowing exposes the seeds to low soil temperatures which could destroy the seed integrity and reduce the germination rate of sorghum (Harris, 1996). Late sowing exposes the plants to drought risks and destroys the normal growth and development of plants that reduces the yield and quality of grain in sorghum (Petrini et al., 1993). Thus, the purpose of sowing time research is to 
increase grain yield and quality of sorghum by ensuring that the germination rate and accumulated temperature needed for growth, and development of plants are adequate. Reasonable growing density is an important prerequisite for high yield and good quality of sorghum (Berenguer \& Faci, 2001; Marsalis et al., 2010; Wang et al., 2013). Low growing density results in big spike and high grain weight per plant because of large nutrient area per plant, full development of single spike, and good ventilation and light transmittance (Carmi et al., 2006; Godsey et al., 2011). However, low growing density leads to some problems such as low grain yield per unit area due to less plant number per unit area and insufficient photosynthesis area (Al-Bedairy et al., 2013). Conversely, high growing density leads to fierce competition among plants and increases the potential for cooperation, thus creating usable differences in the performance of individuals and groups, and results in low grain yield and poor grain quality because of poor ventilation and light transmittance, small nutrient area per plant, and underdeveloped single spike (Mosavi et al., 2009; Wang et al., 2016). Therefore, reasonable growing density can coordinate the relationship between groups and individuals, make individuals grow strong without premature aging, and ensure a certain number of population, which is advantageous to increase the potentials of grain yield and quality in sorghum.

Numerous studies have reported that sowing time and growing density exerts remarkable impacts on agronomic traits, grain yield, and grain quality of sweet and feed sorghum (Almodares \& Mostafafi Darany, 2006; Carmi et al., 2006; Marsalis et al., 2010; Wang et al., 2013). Nevertheless, little is known about the effects of sowing time and growing density on agronomic traits, grain yield, and grain quality of grain sorghum, especially on the waxy sorghum for brewing Moutai flavor liquor. The purpose of this study was to assess the effects of sowing time and growing density on agronomic traits, grain yield, and grain quality and to determine the suitable sowing time and growing density for high yield and good quality in waxy sorghum.

\section{Materials and Methods}

\subsection{Experimental Location}

Field experiments were carried out during 2016 and 2017 in Haohuahong Village (26 ${ }^{\circ} 18^{\prime} \mathrm{N}$ and $\left.106^{\circ} 22^{\prime} \mathrm{E}\right)$, Haohuahong Town, Huishui County, Qiannan State, Guizhou Province, China. The village is located $85 \mathrm{~km}$ south of Guizhou Academy of Agricultural Sciences at an elevation of $1050 \mathrm{~m}$ above sea level. Annual mean temperature is $16.20{ }^{\circ} \mathrm{C}$. Annual precipitation is $1250 \mathrm{~mm}$. The frost-free period is 290 days. The region has a subtropical humid temperate climate.

\subsection{Experimental Design}

Hongliangfeng 1, a waxy sorghum cultivar specially used to brew Moutai flavor liquor, which was authorized by the Guizhou Provincial Crop Cultivar Certification Committee (Guiyang, Guizhou, China) in 2016. It is widely grown in the sorghum growing region of Guizhou province.

Field experiments were conducted using a split plot design and replicated three times. The main plots were two sowing time: 5 April (early sowing, S1) and 20 April (late sowing, S2). The subplots were three growing density: $0.8 \times 10^{5}$ plants/ha (low, P1), $1.1 \times 10^{5}$ plants/ha (usual, P2), and $1.4 \times 10^{5}$ plants/ha (high, P3). Each plot was 9 $\mathrm{m}$ long, $3.5 \mathrm{~m}$ wide, about $70 \mathrm{~cm}$ apart, and consisted of five rows. There were three protected rows around each plot. Compound fertilizer (containing $15 \% \mathrm{~N}, 15 \% \mathrm{P}_{2} \mathrm{O}_{5}$, and $15 \% \mathrm{~K}_{2} \mathrm{O}$ ) was used as a basal fertilizer at a dose of $450 \mathrm{~kg} / \mathrm{ha}$ at sowing time. Final singling was performed at the three-leaf stage. Urea (containing $46.4 \% \mathrm{~N}$ ) was used as additional fertilizer at a dose of $300 \mathrm{~kg} / \mathrm{ha}$ at the jointing stage. The prevention measures of fungicides, pesticides, herbicides were applied to prevent diseases, pests, and grasses in both seasons. No significant occurrence of diseases, pests, or grasses and no impacts on sorghum growth were found in both seasons.

\subsection{Determination of Agronomic Traits, Grain yield, and Grain quality}

Plant height, spike length, and culm diameter were measured at three days before harvest. Five plants were selected randomly from each plot, excluding the guard rows. Plant height was measured as distance from the base of culm to the top of plant and spike length as distance from the base of spike to the top of spike. Culm diameter refers to the diameter of the 2 nd internode at the bottom, and was determined by a vernier caliper (Forgestar 7D-01150, Shanghai, China). At maturity, all plants were hand-harvested. Grain number per spike was measured with an automatic particle meter (Dacheng SLY-C, Hangzhou, China). For grain weight per plant, 1000-grain weight, and grain yield, seeds were determined after being dried for seven days.

Seed samples were refrigerated until grain quality analyses were conducted. The contents of protein, tannin, starch, and amylopectin were determined using a FOSS grain quality analyzer (NIRSystem TR-3750, Shanghai, 
China) according to the method of Xiao et al. (2004). The amylose content was calculated as starch content minus amylopectin content.

\subsection{Data Analyses}

Data collected were entered in Microsoft Excel 2013. Analysis of variance was conducted by DPS v3.01. Significant means were separated by LSD method. Figures were drawn by SigmaPlot 10.0.

\section{Results}

\subsection{Basic Information of Experimental Site}

The soil was silty loam with a $\mathrm{pH}$ of 7.68 and rape (Brassica napus L.) was the preceding crop. The organic content was $33.68 \mathrm{~g} / \mathrm{kg}$, the total $\mathrm{N}$, P, and $\mathrm{K}$ concentrations were $1.27,1.08$, and $9.54 \mathrm{~g} / \mathrm{kg}$, and the available $\mathrm{N}, \mathrm{P}$, and $\mathrm{K}$ concentrations were $123.36,46.33$, and $437.65 \mathrm{mg} / \mathrm{kg}$ in the $0-200 \mathrm{~mm}$ soil horizon, respectively. The meteorological data during the growing period are shown in Figure 1.

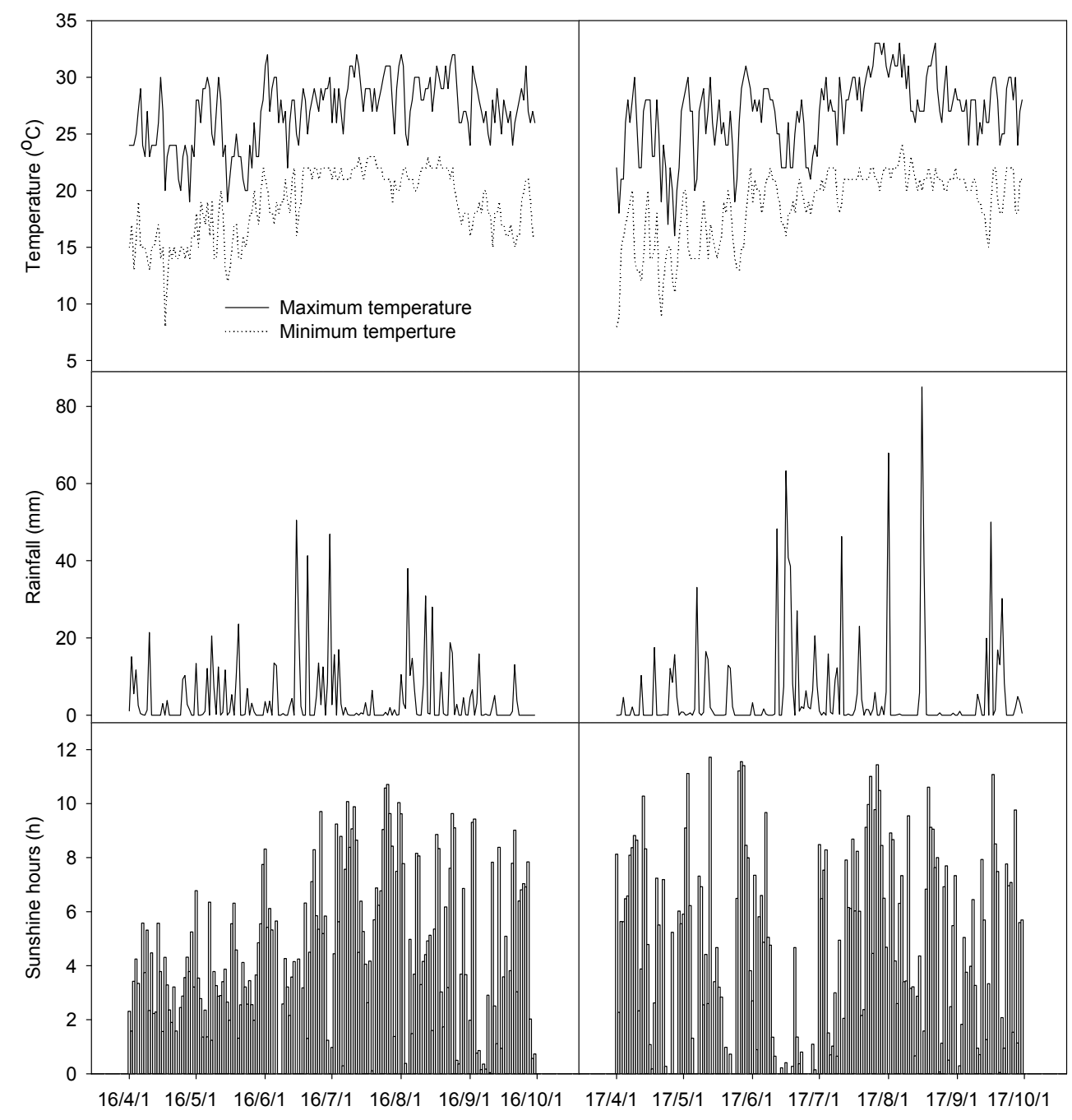

Figure 1. Climate data during the experimental period

\subsection{Grain Yield}

Grain yield was significantly influenced by sowing time and growing density, but the interactions were not significant in the two years (Table 1). Single factor analysis (Table 2) showed that the grain yield in early sowing was higher than that in late sowing. Compared with late sowing, early sowing increased grain yield by $11.42 \%$ in 2016 and $11.53 \%$ in 2017 . Grain yield significantly increased and then reduced with the increase of the growing 
density in two years. Interaction between sowing time and growing density (Table 3 ) showed that the early sowing and growing density of $1.1 \times 10^{5}$ plants/ha treatments had the highest grain yield in 2016 and 2017 .

\subsection{Agronomic Traits}

As shown by variance analysis (Table 1), sowing time had significant effect on spike length, culm diameter, grain number per spike, and grain number per plant, while it had no remarkable effect on the plant height and 1000 -grain weight in both years. Growing density had significant effect on all agronomic traits in two years. Interaction between sowing time and growing density had significant effect on grain number per spike and grain number per plant, but had no significant effect on plant height, spike length, culm diameter, and 1000-grain weight in both years.

Table 1. Variance analysis for effects of growing density and sowing time on agronomic traits and grain yield in waxy sorghum cultivar Hongliangfeng 1 ( $F$-value)

\begin{tabular}{|c|c|c|c|c|c|c|c|c|c|}
\hline Year & $\begin{array}{l}\text { Source of } \\
\text { variation }\end{array}$ & $\begin{array}{l}\text { Degree of } \\
\text { freedom }\end{array}$ & Grain yield & Plant height & Spike length & Culm diameter & $\begin{array}{l}\text { Grain number } \\
\text { per spike }\end{array}$ & $\begin{array}{l}\text { Grain weight } \\
\text { per plant }\end{array}$ & $\begin{array}{l}\text { 1000-grain } \\
\text { weight }\end{array}$ \\
\hline \multirow{3}{*}{2016} & $S$ & 1 & $760.92 * *$ & $0.03 \mathrm{~ns}$ & $47.48 *$ & $38.11 *$ & $23.24 *$ & $20.32 *$ & $11.87 \mathrm{~ns}$ \\
\hline & $\mathrm{P}$ & 2 & $7.34 *$ & $5.62 *$ & $13.45 * *$ & $17.44 * *$ & $30.99 * *$ & $187.02 * *$ & $87.26 * *$ \\
\hline & $\mathrm{S} \times \mathrm{P}$ & 2 & $1.32 \mathrm{~ns}$ & $1.43 \mathrm{~ns}$ & $0.08 \mathrm{~ns}$ & $0.61 \mathrm{~ns}$ & $13.46 * *$ & $32.78 * *$ & $0.02 \mathrm{~ns}$ \\
\hline \multirow{3}{*}{2017} & $\mathrm{~S}$ & 1 & $200.68 * *$ & $0.01 \mathrm{~ns}$ & $47.32 *$ & $45.24 *$ & $229.76 * *$ & $50.96 * *$ & $4.96 \mathrm{~ns}$ \\
\hline & $\mathrm{P}$ & 2 & $24.34 * *$ & $6.02 *$ & $63.69 * *$ & $62.16 * *$ & $44.57 * *$ & $142.33 * *$ & $28.16 * *$ \\
\hline & $\mathrm{S} \times \mathrm{P}$ & 2 & $2.80 \mathrm{~ns}$ & $2.16 \mathrm{~ns}$ & $1.84 \mathrm{~ns}$ & $2.92 \mathrm{~ns}$ & $10.26 * *$ & $10.96 * *$ & $1.41 \mathrm{~ns}$ \\
\hline
\end{tabular}

Note. $\mathrm{S}, \mathrm{P}$, and $\mathrm{S} \times \mathrm{P}$ represent sowing time, growing density, and interaction between sowing time and growing density, respectively. ${ }^{*}$ and ${ }^{* *}$ represent significant at $P<0.05$ and $P<0.01$, respectively. ns represent not significant.

Table 2. Effects of growing density and sowing time on agronomic traits and grain yield in waxy sorghum cultivar Hongliangfeng 1

\begin{tabular}{lllllllll}
\hline Year & Treatments & $\begin{array}{l}\text { Grain yield } \\
(\mathrm{kg} / \mathrm{ha})\end{array}$ & $\begin{array}{l}\text { Plant height } \\
(\mathrm{cm})\end{array}$ & $\begin{array}{l}\text { Spike length } \\
(\mathrm{cm})\end{array}$ & $\begin{array}{l}\text { Culm diameter } \\
(\mathrm{mm})\end{array}$ & $\begin{array}{l}\text { Grain number } \\
\text { per spike }\end{array}$ & $\begin{array}{l}\text { Grain weight } \\
\text { per plant }(\mathrm{g})\end{array}$ & $\begin{array}{l}1000 \text {-grain } \\
\text { weight }(\mathrm{g})\end{array}$ \\
\hline \multirow{6}{*}{2016} & $\mathrm{~S} 1$ & $5517.57 \mathrm{a}$ & $209.75 \mathrm{a}$ & $33.15 \mathrm{a}$ & $14.56 \mathrm{a}$ & $4024.13 \mathrm{a}$ & $89.13 \mathrm{a}$ & $22.24 \mathrm{a}$ \\
& $\mathrm{S} 2$ & $4952.21 \mathrm{~b}$ & $208.86 \mathrm{a}$ & $32.24 \mathrm{~b}$ & $11.95 \mathrm{~b}$ & $3754.18 \mathrm{~b}$ & $83.52 \mathrm{~b}$ & $21.30 \mathrm{a}$ \\
& $\mathrm{P} 1$ & $5093.82 \mathrm{~b}$ & $199.92 \mathrm{~b}$ & $30.33 \mathrm{c}$ & $12.02 \mathrm{~b}$ & $3822.77 \mathrm{~b}$ & $83.30 \mathrm{~b}$ & $20.70 \mathrm{c}$ \\
& P2 & $5486.08 \mathrm{a}$ & $220.30 \mathrm{a}$ & $35.13 \mathrm{a}$ & $14.94 \mathrm{a}$ & $4061.72 \mathrm{a}$ & $94.15 \mathrm{a}$ & $22.96 \mathrm{a}$ \\
& P3 & $5124.78 \mathrm{~b}$ & $199.92 \mathrm{~b}$ & $32.62 \mathrm{~b}$ & $12.81 \mathrm{~b}$ & $3783.00 \mathrm{~b}$ & $81.53 \mathrm{c}$ & $21.79 \mathrm{~b}$ \\
\hline \multirow{4}{*}{2017} & S1 & $5692.24 \mathrm{a}$ & $211.65 \mathrm{a}$ & $33.44 \mathrm{a}$ & $14.90 \mathrm{a}$ & $4093.12 \mathrm{a}$ & $92.31 \mathrm{a}$ & $21.96 \mathrm{a}$ \\
& S2 & $5103.63 \mathrm{~b}$ & $211.12 \mathrm{a}$ & $30.90 \mathrm{~b}$ & $12.50 \mathrm{~b}$ & $3798.30 \mathrm{~b}$ & $86.17 \mathrm{~b}$ & $20.94 \mathrm{a}$ \\
& P1 & $5207.38 \mathrm{~b}$ & $204.79 \mathrm{~b}$ & $30.35 \mathrm{c}$ & $12.48 \mathrm{c}$ & $3848.74 \mathrm{~b}$ & $86.15 \mathrm{~b}$ & $20.18 \mathrm{c}$ \\
& P2 & $5717.76 \mathrm{a}$ & $222.48 \mathrm{a}$ & $34.11 \mathrm{a}$ & $15.30 \mathrm{a}$ & $4118.98 \mathrm{a}$ & $98.68 \mathrm{a}$ & $23.01 \mathrm{a}$ \\
& P3 & $5268.66 \mathrm{~b}$ & $206.88 \mathrm{~b}$ & $32.05 \mathrm{~b}$ & $13.32 \mathrm{~b}$ & $3869.41 \mathrm{~b}$ & $82.89 \mathrm{c}$ & $21.17 \mathrm{~b}$
\end{tabular}

Note. Data are expressed as the mean of three replications $(n=3)$. Values followed by different letters within a column represent significantly different at $P<0.05$. S1 and S2 represent sowing time at two levels of 5 April and 20 April, respectively. P1, P2, and P3 represent growing density at three levels of $0.8 \times 10^{5}, 1.1 \times 10^{5}$, and $1.4 \times$ $10^{5}$ plants/ha, respectively.

Single factor analysis (Table 2) showed that there were no significant differences in plant height and 1000-grain weight between early and late sowing in both years. Spike length, culm diameter, grain number per spike, and grain number per plant were higher than those in late sowing. Compared with late sowing, early sowing markedly increased the spike length, culm diameter, grain number per spike, and grain number per plant by $5.46 \%, 20.49 \%, 7.48 \%$, and $6.92 \%$ (mean of 2016 and 2017), respectively. Plant height, spike length, culm diameter, grain number per spike, grain number per plant, and 1000- grain weight increased and then reduced with the increase in growing density in two years. Interaction between sowing time and growing density (Table 3) showed that early sowing and growing density of $1.1 \times 10^{5}$ plants/ha treatments had the highest plant height, 
spike length, culm diameter, grain number per spike, grain number per plant, and 1000-grain weight in both years.

Table 3. Effects of interaction between sowing time and growing density on agronomic traits and grain yield in waxy sorghum Hongliangfeng 1

\begin{tabular}{lllllllll}
\hline Year & Treatments & $\begin{array}{l}\text { Grain yield } \\
(\mathrm{kg} / \mathrm{ha})\end{array}$ & $\begin{array}{l}\text { Plant height } \\
(\mathrm{cm})\end{array}$ & $\begin{array}{l}\text { Spike length } \\
(\mathrm{cm})\end{array}$ & $\begin{array}{l}\text { Culm diameter } \\
(\mathrm{mm})\end{array}$ & $\begin{array}{l}\text { Grain number } \\
\text { per spike }\end{array}$ & $\begin{array}{l}\text { Grain weight } \\
\text { per plant }(\mathrm{g})\end{array}$ & $\begin{array}{l}\text { 1000-grain } \\
\text { weight }(\mathrm{g})\end{array}$ \\
\hline & $\mathrm{S} 1 \times \mathrm{P} 1$ & $5447.17 \mathrm{~b}$ & $195.16 \mathrm{c}$ & $30.69 \mathrm{bc}$ & $13.26 \mathrm{bc}$ & $3982.35 \mathrm{~b}$ & $89.04 \mathrm{~b}$ & $21.21 \mathrm{c}$ \\
& $\mathrm{S} 1 \times \mathrm{P} 2$ & $5802.90 \mathrm{a}$ & $225.89 \mathrm{a}$ & $35.48 \mathrm{a}$ & $16.55 \mathrm{a}$ & $4281.41 \mathrm{a}$ & $96.80 \mathrm{a}$ & $23.50 \mathrm{a}$ \\
2016 & $\mathrm{~S} 1 \times \mathrm{P} 3$ & $5302.90 \mathrm{~b}$ & $208.20 \mathrm{abc}$ & $33.29 \mathrm{ab}$ & $13.87 \mathrm{~b}$ & $3808.63 \mathrm{c}$ & $81.55 \mathrm{c}$ & $22.30 \mathrm{~b}$ \\
& $\mathrm{~S} 2 \times \mathrm{P} 1$ & $4740.46 \mathrm{~d}$ & $204.69 \mathrm{bc}$ & $29.98 \mathrm{c}$ & $10.77 \mathrm{~d}$ & $3663.18 \mathrm{~d}$ & $77.56 \mathrm{~d}$ & $20.19 \mathrm{~d}$ \\
& $\mathrm{~S} 2 \times \mathrm{P} 2$ & $5169.25 \mathrm{bc}$ & $214.70 \mathrm{ab}$ & $34.79 \mathrm{a}$ & $13.32 \mathrm{bc}$ & $3842.03 \mathrm{bc}$ & $91.51 \mathrm{~b}$ & $22.42 \mathrm{~b}$ \\
& $\mathrm{~S} 2 \times \mathrm{P} 3$ & $4946.92 \mathrm{~cd}$ & $207.20 \mathrm{abc}$ & $31.95 \mathrm{bc}$ & $11.75 \mathrm{~cd}$ & $3757.34 \mathrm{~cd}$ & $81.50 \mathrm{c}$ & $21.29 \mathrm{c}$ \\
\hline \multirow{2}{*}{2017} & $\mathrm{~S} 1 \times \mathrm{P} 1$ & $5508.95 \mathrm{~b}$ & $200.78 \mathrm{~b}$ & $31.26 \mathrm{c}$ & $13.91 \mathrm{~b}$ & $4005.92 \mathrm{~b}$ & $91.31 \mathrm{c}$ & $20.43 \mathrm{~cd}$ \\
& $\mathrm{~S} 1 \times \mathrm{P} 2$ & $6102.82 \mathrm{a}$ & $229.34 \mathrm{a}$ & $35.47 \mathrm{a}$ & $16.62 \mathrm{a}$ & $4333.17 \mathrm{a}$ & $102.14 \mathrm{a}$ & $23.88 \mathrm{a}$ \\
& $\mathrm{S} 1 \times \mathrm{P} 3$ & $5464.96 \mathrm{~b}$ & $204.83 \mathrm{~b}$ & $33.58 \mathrm{~b}$ & $14.16 \mathrm{~b}$ & $3940.29 \mathrm{bc}$ & $83.47 \mathrm{~d}$ & $21.57 \mathrm{bc}$ \\
& $\mathrm{S} 2 \times \mathrm{P} 1$ & $4905.82 \mathrm{c}$ & $208.82 \mathrm{~b}$ & $29.44 \mathrm{~d}$ & $11.05 \mathrm{~d}$ & $3691.57 \mathrm{e}$ & $80.99 \mathrm{~d}$ & $19.92 \mathrm{~d}$ \\
& $\mathrm{~S} 2 \times \mathrm{P} 2$ & $5332.71 \mathrm{~b}$ & $215.61 \mathrm{ab}$ & $32.75 \mathrm{~b}$ & $13.98 \mathrm{~b}$ & $3904.79 \mathrm{c}$ & $95.22 \mathrm{~b}$ & $22.14 \mathrm{~b}$ \\
& $\mathrm{~S} 2 \times \mathrm{P} 3$ & $5072.36 \mathrm{c}$ & $208.93 \mathrm{~b}$ & $30.52 \mathrm{~cd}$ & $12.48 \mathrm{c}$ & $3798.51 \mathrm{~d}$ & $82.30 \mathrm{~d}$ & $20.76 \mathrm{~cd}$ \\
\hline
\end{tabular}

Note. Data are expressed as the mean of three replications $(n=3)$. Values followed by different letters within a column represent significantly different at $P<0.05$. S1 and S2 represent sowing time at two levels of 5 April and 20 April, respectively. P1, P2, and P3 represent growing density at three levels of $0.8 \times 10^{5}, 1.1 \times 10^{5}$, and $1.4 \times$ $10^{5}$ plants/ha, respectively.

\subsection{Grain Quality}

Sowing time had significant effect on protein content in the two years. Growing density and interaction between sowing time and growing density had significant effects on protein content in 2017, while it had no effects on protein content in 2016 (Table 4). As shown by single factor analysis (Table 5), protein content in early sowing was higher than that in late sowing. Compared with late sowing, early sowing significantly increased protein content by $4.83 \%$ in 2016 and $9.49 \%$ in 2017 . There were no significant differences in protein content among the three growing densities in 2016. Protein content increased and then reduced with the increase in growing density in 2017. Interaction between sowing time and growing density (Table 6) showed that early sowing and growing density of $1.1 \times 10^{5}$ plants/ha treatments had the highest protein content in the two years.

Table 4. Variance analysis for effects of growing density and sowing time on grain quality in waxy sorghum cultivar Hongliangfeng 1 ( $F$-value)

\begin{tabular}{|c|c|c|c|c|c|c|c|}
\hline Year & $\begin{array}{l}\text { Source of } \\
\text { variation }\end{array}$ & $\begin{array}{l}\text { Degree of } \\
\text { freedom }\end{array}$ & Protein content & Tannin content & Starch content & Amylopectin content & Amylose content \\
\hline \multirow{3}{*}{2016} & $\mathrm{~S}$ & 1 & $27.14 *$ & $43.73 *$ & $13.60 \mathrm{~ns}$ & $37.59 *$ & $135.32 * *$ \\
\hline & $\mathrm{P}$ & 2 & $1.37 \mathrm{~ns}$ & $654.42 * *$ & $29.73 * *$ & $70.20 * *$ & $2.36 \mathrm{~ns}$ \\
\hline & $\mathrm{S} \times \mathrm{P}$ & 2 & $0.63 \mathrm{~ns}$ & $16.44 * *$ & $1.49 \mathrm{~ns}$ & $2.12 \mathrm{~ns}$ & $2.54 \mathrm{~ns}$ \\
\hline \multirow{3}{*}{2017} & $\mathrm{~S}$ & 1 & $40.17 *$ & $36.33 *$ & $29.64 *$ & $9.92 \mathrm{~ns}$ & $6.11 \mathrm{~ns}$ \\
\hline & $\mathrm{P}$ & 2 & $115.01 * *$ & $101.59 * *$ & $116.55 * *$ & $45.49 * *$ & $4.76 *$ \\
\hline & $\mathrm{S} \times \mathrm{P}$ & 2 & $42.59 * *$ & $12.40 * *$ & $4.71 *$ & $0.37 \mathrm{~ns}$ & $2.39 \mathrm{~ns}$ \\
\hline
\end{tabular}

Note. $\mathrm{S}, \mathrm{P}$, and $\mathrm{S} \times \mathrm{P}$ represent sowing time, growing density, and interaction between sowing time and growing density, respectively. ${ }^{*}$ and ${ }^{* *}$ represent significant at $P<0.05$ and $P<0.01$, respectively. ns represent not significant.

Tannin content was significantly influenced by sowing time, growing density, and their interaction in the two years (Table 4). Single factor analysis (Table 5) showed that tannin content in early sowing was lower than that in late sowing. Compared with late sowing, early sowing significantly decreased the tannin content by $7.71 \%$ in 
2016 and $8.48 \%$ in 2017. Tannin content reduced and then increased with the increase of growing density in both years. Interaction between sowing time and growing density (Table 6) showed that early sowing and growing density of $1.1 \times 10^{5}$ plants/ha treatments had the lowest tannin content in the two years.

Growing density had significant effect on starch content in the two years. Sowing time and interaction between sowing time and growing density had no significant effects on starch content in 2016, while it had significant effects on starch content in 2017 (Table 4). Single factor analysis (Table 5) showed no significant differences in starch content between early and late sowing in 2016. Early sowing increased the starch content by $1.08 \%$ compared with late sowing in 2017. Starch content increased and then reduced with the increase in growing density in both years. Interaction between sowing time and growing density (Table 6) showed that early sowing and growing density of $1.1 \times 10^{5}$ plants/ha treatments had the highest starch content in the two years.

Table 5. Effects of growing density and sowing time on grain quality in waxy sorghum cultivar Hongliangfeng 1

\begin{tabular}{lllllll}
\hline Year & Treatments & $\begin{array}{l}\text { Protein content } \\
(\%)\end{array}$ & $\begin{array}{l}\text { Tannin content } \\
(\%)\end{array}$ & $\begin{array}{l}\text { Starch content } \\
(\%)\end{array}$ & $\begin{array}{l}\text { Amylopectin content } \\
(\%)\end{array}$ & $\begin{array}{l}\text { Amylose content } \\
(\%)\end{array}$ \\
\hline \multirow{6}{*}{2016} & S1 & $11.40 \mathrm{a}$ & $1.46 \mathrm{~b}$ & $80.09 \mathrm{a}$ & $72.44 \mathrm{a}$ & $7.65 \mathrm{~b}$ \\
& $\mathrm{~S} 2$ & $10.87 \mathrm{~b}$ & $1.59 \mathrm{a}$ & $79.33 \mathrm{a}$ & $70.68 \mathrm{~b}$ & $8.65 \mathrm{a}$ \\
& P1 & $11.01 \mathrm{a}$ & $1.54 \mathrm{~b}$ & $78.35 \mathrm{c}$ & $69.82 \mathrm{c}$ & $8.53 \mathrm{a}$ \\
& P2 & $11.24 \mathrm{a}$ & $1.41 \mathrm{c}$ & $80.84 \mathrm{a}$ & $73.27 \mathrm{a}$ & $7.57 \mathrm{a}$ \\
& P3 & $11.16 \mathrm{a}$ & $1.63 \mathrm{a}$ & $79.94 \mathrm{~b}$ & $71.59 \mathrm{~b}$ & $8.36 \mathrm{a}$ \\
\hline \multirow{2}{*}{2017} & $\mathrm{~S} 1$ & $11.95 \mathrm{a}$ & $1.38 \mathrm{~b}$ & $80.40 \mathrm{a}$ & $72.88 \mathrm{a}$ & $7.96 \mathrm{a}$ \\
& S2 & $10.91 \mathrm{~b}$ & $1.51 \mathrm{a}$ & $79.54 \mathrm{~b}$ & $71.51 \mathrm{a}$ & $8.86 \mathrm{a}$ \\
& P1 & $11.06 \mathrm{~b}$ & $1.47 \mathrm{~b}$ & $78.81 \mathrm{c}$ & $70.36 \mathrm{c}$ & $8.98 \mathrm{a}$ \\
& P2 & $12.06 \mathrm{a}$ & $1.32 \mathrm{c}$ & $81.12 \mathrm{a}$ & $73.97 \mathrm{a}$ & $7.85 \mathrm{~b}$ \\
& P3 & $11.17 \mathrm{~b}$ & $1.54 \mathrm{a}$ & $79.98 \mathrm{~b}$ & $72.26 \mathrm{~b}$ & $8.39 \mathrm{ab}$ \\
\hline
\end{tabular}

Note. Data are expressed as the mean of three replications $(n=3)$. Values followed by different letters within a column represent significantly different at $P<0.05$. S1 and S2 represent sowing time at two levels of 5 April and 20 April, respectively. P1, P2, and P3 represent growing density at three levels of $0.8 \times 10^{5}, 1.1 \times 10^{5}$, and $1.4 \times$ $10^{5}$ plants/ha, respectively.

Sowing time had significant influence on amylopectin content in 2016, but it had no significant influence in 2017. Growing density had significant influence on amylopectin content in both years. Interaction between sowing time and growing density had no significant influences on amylopectin content in the two years (Table 4). Compared with late sowing, early sowing increased the amylopectin content by $2.49 \%$ in 2016 , while it had no differences in amylopectin content between early and late sowing in 2017 (Table 5). Amylopectin content increased and then reduced with the increase in growing density in both years. Interaction between sowing time and growing density (Table 6) showed that early sowing and growing density of $1.1 \times 10^{5}$ plants/ha treatments had the highest amylopectin content in the two years.

Sowing time had significant influence on amylose content in 2016, but it had no significant influence in 2017. Growing density had no significant influence on amylose content in 2016, but it had significant influence in 2017. Interaction between sowing time and growing density had no significant influences on amylose content in the two years (Table 4). Compared with late sowing, early sowing decreased amylose content by $11.63 \%$ in 2016 , while it no differences in amylose content were found in 2017 (Table 5). There were no significant differences in amylose content among the three growing densities in 2016. Amylose content reduced and then increased with the increase in growing density in 2017. Interaction between sowing time and growing density (Table 6) showed that early sowing and growing density of $1.1 \times 10^{5}$ plants/ha treatments had the lowest amylose content in the two years. 
Table 6. Effects of interaction between growing density and sowing time on grain quality in waxy sorghum cultivar Hongliangfeng 1

\begin{tabular}{lllllll}
\hline Year & Treatments & $\begin{array}{l}\text { Protein content } \\
(\%)\end{array}$ & $\begin{array}{l}\text { Tannin content } \\
(\%)\end{array}$ & $\begin{array}{l}\text { Starch content } \\
(\%)\end{array}$ & $\begin{array}{l}\text { Amylopectin content } \\
(\%)\end{array}$ & $\begin{array}{l}\text { Amylose content } \\
(\%)\end{array}$ \\
\hline \multirow{2}{*}{2016} & $\mathrm{~S} 1 \times \mathrm{P} 1$ & $11.35 \mathrm{ab}$ & $1.47 \mathrm{c}$ & $78.82 \mathrm{c}$ & $70.40 \mathrm{c}$ & $8.43 \mathrm{abc}$ \\
& $\mathrm{S} 1 \times \mathrm{P} 2$ & $11.43 \mathrm{a}$ & $1.34 \mathrm{~d}$ & $81.44 \mathrm{a}$ & $74.17 \mathrm{a}$ & $7.27 \mathrm{c}$ \\
& $\mathrm{S} 1 \times \mathrm{P} 3$ & $11.42 \mathrm{a}$ & $1.58 \mathrm{~b}$ & $80.01 \mathrm{~b}$ & $72.76 \mathrm{~b}$ & $7.25 \mathrm{c}$ \\
& $\mathrm{S} 2 \times \mathrm{P} 1$ & $10.66 \mathrm{c}$ & $1.61 \mathrm{~b}$ & $77.88 \mathrm{c}$ & $69.25 \mathrm{~d}$ & $8.63 \mathrm{ab}$ \\
& $\mathrm{S} 2 \times \mathrm{P} 2$ & $11.06 \mathrm{abc}$ & $1.48 \mathrm{c}$ & $80.23 \mathrm{~b}$ & $72.37 \mathrm{~b}$ & $7.86 \mathrm{bc}$ \\
& $\mathrm{S} 2 \times \mathrm{P} 3$ & $10.91 \mathrm{bc}$ & $1.67 \mathrm{a}$ & $79.88 \mathrm{~b}$ & $70.42 \mathrm{c}$ & $9.46 \mathrm{a}$ \\
& $\mathrm{S} 1 \times \mathrm{P} 1$ & $11.87 \mathrm{ab}$ & $1.36 \mathrm{c}$ & $79.14 \mathrm{~d}$ & $70.85 \mathrm{~cd}$ & $8.75 \mathrm{abc}$ \\
& $\mathrm{S} 1 \times \mathrm{P} 2$ & $12.21 \mathrm{a}$ & $1.29 \mathrm{c}$ & $81.81 \mathrm{a}$ & $74.76 \mathrm{a}$ & $7.64 \mathrm{~cd}$ \\
& $\mathrm{~S} 1 \times \mathrm{P} 3$ & $11.75 \mathrm{~b}$ & $1.48 \mathrm{~b}$ & $80.24 \mathrm{~b}$ & $73.03 \mathrm{~b}$ & $7.48 \mathrm{~d}$ \\
& $\mathrm{~S} 2 \times \mathrm{P} 1$ & $10.24 \mathrm{c}$ & $1.57 \mathrm{a}$ & $78.47 \mathrm{e}$ & $69.86 \mathrm{~d}$ & $9.22 \mathrm{ab}$ \\
& $\mathrm{S} 2 \times \mathrm{P} 2$ & $11.90 \mathrm{ab}$ & $1.35 \mathrm{c}$ & $80.43 \mathrm{~b}$ & $73.18 \mathrm{~b}$ & $8.06 \mathrm{bcd}$ \\
\hline
\end{tabular}

Note. Data are expressed as the mean of three replications $(n=3)$. Values followed by different letters within a column represent significantly different at $P<0.05$. S1 and S2 represent sowing time at two levels of 5 April and 20 April, respectively. P1, P2, and P3 represent growing density at three levels of $0.8 \times 10^{5}, 1.1 \times 10^{5}$, and $1.4 \times$ $10^{5}$ plants/ha, respectively.

\subsection{Correlation Analysis Among Grain Yield, Amylopectin Content, and Grain Yield Components}

Grain yield was significantly and positively correlated with grain number per spike, grain weight per plant, 1000-grain weight, and amylopectin content (Table 7). Similarly, amylopectin content was significantly and positively correlated with grain number per spike, grain weight per plant, and 1000-grain weight.

Table 7. Correlation coefficients among grain yield, amylopectin content, and grain yield components in waxy sorghum cultivar Hongliangfeng 1

\begin{tabular}{lllll}
\hline & Amylopectin content & Grain number per spike & Grain weight per plant & 1000-grain weight \\
\hline Amylopectin content & & $0.761^{* *}$ & $0.738^{* *}$ & $0.920^{* *}$ \\
Grain yield & $0.818^{* *}$ & $0.974^{* *}$ & $0.856^{* *}$ & $0.778^{* *}$ \\
\hline
\end{tabular}

Note. ${ }^{* *}$ represent significantly correlation at $P<0.01$.

\section{Discussion}

Sorghum yield is determined by spike number per growing area, grain number per spike, grain weight per plant, and 1000-grain weight. The spike number per growing area mainly reflects the changing amplitude of population density and the grain number per spike, grain weight per plant, and 1000-grain weight mainly reflect the growth and development status of individuals within a population (Zhou et al., 2016; Zhou et al., 2017). Generally speaking, amylopectin content is the most critical factor for quality assessment of waxy sorghum cultivars grown for liquor production (Cao, 2016; Wang et al., 2017a). In this study, grain yield and amylopectin content were significantly and positively correlated with grain number per spike, grain weight per plant, and 1000-grain weight, which is consistent with reported by Liu et al. (2012). Therefore, greatly expanding the plant's storage capacity (increasing of grain number per spike, grain weight per plant, and 1000-grain weight) is the premise to realize the high yield and good quality of Hongliangfeng 1 .

Suitable sowing time is one of the key cultivation measures in sorghum production. As reported by Ekeleme et al. (2011), and Luo et al. (2013), in sorghum, yield and quality are strongly reduced by a delay of sowing time. Similar to our study, compared with early sowing (5 April), late sowing (20 April) significantly decreased the grain yield, spike length, culm diameter, grain number per spike, grain weight per spike, protein content, starch content, and amylopectin content, while significantly increased the tannin content and amylose content. There are two reasons that could be responsible for the decrease in the mean values of agronomic traits, grain yield, and grain quality. The first is that sorghum seeds would have low germination if sown late. The second reason is that 
late sowing destroys the normal growth and development of sorghum plants due to high temperatures and rainfall occur during later growth development period (Figure 1). In the present study, there were no significant differences in plant height and 1000-grain weight between early and late sowing in both years. However, Yang et al. (2017) concluded that sowing time had significant effects on the plant height and 1000-grain weight, and the plant height and 1000-grain weight were reduced by a delay of sowing time. The reason for this difference requires further study.

Growing density is one of the important factors to coordinate the contradiction between crop groups and individuals. In general, an increase in the number of plants can make the most of the crop grain yield and grain quality reaches a certain limit or threshold, after that, the growing density of further increase or maintain the same output and quality, either to lower yield and quality (Watanabe et al., 2003; Wang et al., 2009; Fang et al., 2018). Similar to our study, the grain yield, plant height, spike length, culm diameter, grain number per spike, grain weight per plant, 1000-grain weight, protein content, starch content, and amylopectin content increased to their maximums at the growing density of $1.1 \times 10^{5}$ plants/ha, while subsequently decreased at the growing density of $1.4 \times 10^{5}$ plants/ha. However, tannin content and amylose content decreased and then increased with the increase of growing density. This might be due to the weak light irradiance to the leaf by shading and strong competition at high growing density (Fang et al., 2018). Based on the present results, it is suggested that appropriate sowing time and growing density could effectively optimize the population structure, reduce the competition between plants, enhance the grain yield, and improve the quality of waxy sorghum.

\section{Conclusion}

Sowing time and growing density had significant effects on grain yield and grain quality of waxy sorghum cultivar Hongliangfeng 1. Early sowing had a positive on the expression of most agronomic and quality traits. Appropriate growing density need to be identified in order to ensure high yield and good quality in waxy sorghum production.

\section{Acknowledgments}

This study was supported financially by Special Funds for Guizhou Academy of Agricultural Sciences (QNKYYZX2016015), Subject of National Science and Technology Support Plan for the Twelfth Five-Year Plan (2014BAD07B02-2-4), Action Plan of Scientific Research Institutions Service Enterprises in Guizhou (QKHFQ20184005), Special Funds for Central Government Directs Local Science and Technology (QKZYD20184003), and Talent Base for Germplasm Resources Utilization and Innovation of Characteristic Plant in Guizhou (RCJD2018-14).

\section{References}

Al-Bedairy, N. R., Alsaadawi, I. S., \& Shati, R. K. (2013). Combining effect of allelopathic Sorghum bicolor L. (Moench) cultivars with planting densities on companion weeds. Archives of Agronomy and Soil Science, 59, 955-961. https://doi.org/10.1080/03650340.2012.697995

Almodares, A., \& Mostafafi Darany, S. M. (2006). Effects of planting date and time of nitrogen application on yield and sugar content of sweet sorghum. Journal of Environmental Biology, 27, 601-605.

Awika, J. M., Rooney, L. W., Wu, X. L., Prior, R. L., \& Cisneros-Zevallos, L. (2003). Screening methods to measure antioxidant activity of sorghum (Sorghum bicolor) and sorghum Products. Journal of Agricultural and Food Chemistry, 51, 6657-6662. https://doi.org/10.1021/jf034790i

Berenguer, M. J., \& Faci, J. M. (2001). Sorghum (Sorghum bicolor L. Moench) yield compensation processes under different plant densities and variable water supply. European Journal of Agronomy, 15, 43-55. https://doi.org/10.1016/S1161-0301(01)00095-8

Bibi, A., Sadaqat, H. A., Tahir, M. H. N., \& Akram, H. M. (2012). Screen of sorghum (Sorghum bicolor var Moench) for drought tolerance at seedling stage in polyethylene glycol. The Journal of Animal and Plant Sciences, 22, 671-678.

Borrell, A., Mullet, J. E., George-Jaeggli, B., Oosterom, E. J. V., Hammer, G. L., Klein, P. E., \& Jordan, D. R. (2014). Drought adaptation of stay-green sorghum is associated with canopy development, leaf anatomy, root growth, and water uptake. Journal of Experimental Botany, 65, 6251-6263. https://doi.org/10.1093/ jxb/eru232

Cao, X. L. (2002). Correlation of raw materials and liquor-making. Liquor-making Science and Technology, 4, 53-54. 
Carmi, A., Aharoni, Y., Edelstein, M., Umiel, N., Hagiladi, A., Yosef, E., Nikbachat, M., Zenou, A., \& Miron, J. (2006). Effects of irrigation and plant density on yield, composition and in vitro digestibility of a new forage sorghum variety, Tal, at two maturity stages. Animal Feed Science and Technology, 131, 121-133. https://doi.org/10.1016/j.anifeedsci.2006.02.005

Chipanshi, A. C., Chanda, R., \& Totolo, O. (2003). Vulnerability assessment of the maize and sorghum crops to climate change in Botswana. Climatic Change, 61, 339-360. https://oi.org/10.1023/B:CLIM.0000004551. 55871.eb

Ding, G. X., Zhao, G. L., Chen, G. M., Ni, X. L., \& Liu, T. P. (2015). Invention and utilization of glutinous sorghum male sterile line $45 \mathrm{~A}$ with high starch and high combining ability. Agricultural Science and Technology, 16, 486-492.

Ekeleme, F., Kamara, A. Y., Omoigui, L. O., Chikoye, D., Dugje, I. Y., \& Tegbaru, A. (2011). Effect of sowing date on Striga infestation and yield of sorghum (Sorghum bicolor [L.] Moench) cultivars in the Sudan savanna of northeast Nigeria. African Journal of Agricultural Research, 6, 3240-3246.

Fang, X. M., Li, Y. S., Nie, J., Wang, C., Huang, K. H., Zhang, Y. K., ... Yi, Z. L. (2018). Effects of nitrogen fertilizer and planting density on the leaf photosynthetic characteristics, agronomic traits and grain yield in common buckwheat (Fagopyrum esculentum M.). Field Crops Research, 219, 160-168. https://doi.org/ 10.1016/j.fcr.2018.02.001

Godsey, C. B., Linneman, J., Bellmer, D., \& Huhnke, R. (2011). Developing row spacing and planting density recommendations for rainfed sweet sorghum production in the southern plains. Agronomy Journal, 104, 280-286. https://doi.org/10.2134/agronj2011.0289

Han, L. P., Steinberger, Y., Zhao, Y. L., \& Xie, G. H. (2011). Accumulation and partitioning of nitrogen: phosphorus and potassium in different varieties of sweet sorghum. Field Crops Research, 120, 230-240. https://doi.org/10.1016/j.fcr.2010.10.007

Harris, D. (1996). The effects of manure, genotype, seed priming, depth and date of sowing on the emergence and early growth of Sorghum bicolor (L.) Moench in semi-arid Botswana. Soil and Tillage Research, 40, 73-88. https://doi.org/10.1016/S0167-1987(96)01047-1

Jahanzad, E., Jorat, M., Moghadam, H., Sadeghpour, A., Chaichi, M. R., \& Dashtaki, M. (2013). Response of a new and a commonly grown forage sorghum cultivar to limited irrigation and planting density. Agricultural Water Management, 117, 62-69. https://doi.org/10.1016/j.agwat.2012.11.001

Liu, G. F., Bai, W. B., Zhao, J. W., Wang, J. Z., Zheng, X. P., \& Shen, H. Y. (2012). Effects of different planting densities on the agronomic characters and yield of sorghum varieties in medium and late maturity. Journal of Agriculture, 2, 32-35.

Luo, F., Chen, P., Pei, Z. Y., Gao, J. M., \& Sun, S. J. (2013). Effects of sowing date on the biomass and quality of sweet sorghum at different growth stages. Hubei Agricultural Sciences, 52, 3260-3263.

Mace, E. S., Tai, S. S., Gilding, E. K., Li, Y. H., Prentis, P. J., Bian, L. L., ... Wang, J. (2013). Whole-genome sequencing reveals untapped genetic potential in Africa's indigenous cereal crop sorghum. Nature Communications, 4, 2320. https://doi.org/10.1038/ncomms3320

Marsalis, M. A., Angadi, S. V., \& Contreras-Govea, F. E. (2010). Dry matter yield and nutritive value of corn, forage sorghum, and BMR forage sorghum at different plant populations and nitrogen rates. Field Crops Research, 116, 52-57. https://doi.org/10.1016/j.fcr.2009.11.009

Paterson, A. H., Bowers, J. E., Bruggmann, R., Dubchak, I., Grimwood, J., Gundlach, H., ... Rokhsar, D. S. (2009). The Sorghum bicolor genome and the diversification of grasses. Nature, 457, 551-556. https://doi.org/10.1038/nature07723

Peng, Q. (2011). Status and problem and countermeasures of brewing sorghum in Guizhou. Seed, 30, 68-71.

Petrini, C., Belletti, A., \& Salamini, F. (1993). Accumulation and distribution of dry matter and soluble carbohydrates in two sweet sorghum cultivars influence of sowing date and harvesting time. European Journal of Agronomy, 2, 185-192. https://doi.org/10.1016/S1161-0301(14)80128-7

Mosavi, S. G. R., Seghatoleslami, M. J., Javadi, H., \& Ansari-nia, E. (2009). Effect of plant density and planting pattern on yield, yield components and morphological traits of forage sorghum in second cultivation. Plant Ecophysiology, 2, 81-84. 
Townend, J., Mtakwa, P. W., Mullins, C. E., \& Simmonds, L. P. (1996). Soil physical factors limiting establishment of sorghum and cowpea in two contrasting soil types in the semi-arid tropics. Soil and Tillage Research, 40, 89-106. https://doi.org/10.1016/S0167-1987(96)80008-0

Wang, C., Yang, K. J., Wei, J. P., \& Tang, C. S. (2016). The yield and quality of brewing sorghum performance under different fertilizer density factors. Crops, 1, 98-104.

Wang, C., Zhou, L. B., Zhang, G. B., Xu, Y., Zhang, L. Y., Gao, X., ... Shao, M. B. (2017a). Optimal fertilization for high yield and good quality of waxy sorghum (Sorghum bicolor L. Moench). Field Crops Research, 203, 1-7. https://doi.org/10.1016/j.fcr.2016.12.009

Wang, C., Zhou, L. B., Zhang, G. B., Xu, Y., Zhang, L. Y., Gao, X., ... Shao, M. B. (2017b). Drought resistance identification and drought resistance indices screening of liquor-making waxy sorghum resources at adult plant stage. Scientia Agricultura Sinica, 50, 1388-1402.

Wang, J. S., Yang, N., Dong, E. W., Wang, L. G., Wu, A. L., Ding, Y. C., ... Jiao, X. Y. (2013). Effect of different plant density on growth, yield and nutrient uptake of sorghum. Chinese Agricultural Science Bulletin, 29, 253-258.

Wang, R, Liu, G. S., Ni, G. S., Bi, Q. W., Yang, L. B., \& Zhen, C. H. (2009). Effects of planting density on photosynthetic characteristics and assimilate accumulation of leaves in different positions in flue-cured tobacco. Acta Agronomica Sinica, 35, 2288-2295. https://doi.org/10.3724/SP.J.1006.2009.02288

Watanabe, S., Nakano, Y., \& Okano, K. (2003). Effect of planting density on fruit size: light-interception and photosynthetic activity of vertically trained watermelon (Citrullus lanatus (Thunb.) Matsum. et Nakai) plants. Journal of the Japanese Society Horticultural Science, 72, 497-503. https://doi.org/10.2503/ jjshs. 72.497

Xiao, X., Xie, X. H., Chen, Y., Luo, W. Y., Chen, J. W., Liu, Y. Z., ... Li, X. F. (2004). Application of near infrared transmittance spectroscopy (NITS) to the determination of amylose content of rice grain. Scientia Agricultura Sinica, 37, 1709-1712.

Yang, L., Cui, F. Z., Duan, Y. H., Guo, X. Q., Xue, J. F., Cui, J. H., \& Sun, L. X. (2017). Effects of different sowing dates on the growth stages and yield of summer sorghum. Journal of Shanxi Agricultural University (Natural Science Edition), 37, 7-10.

Zhou, K. F., Chen, M., \& Zheng, M. Q. (2008). Occurrence of main disease and pest and its controlling countermeasures in organic sorghum for maotai wine. Guizhou Agricultural Sciences, 36, 90-91.

Zhou, L. B., Wang, C., Lu, X. J., Zhang, G. B., Xu, Y., Wu, L. Y., \& Shao, M. B. (2016). Effects of fertilizer application rate and planting density on photosynthetic characteristics, agronomic traits and yield of waxy sorghum Qiangao 7. Journal of Southern Agriculture, 47, 644-648.

Zhou, L. B., Wang, C., Zhang, G. B., Xu, Y., Bai, J. X., \& Shao, M. B. (2017). Effects of sowing date, planting density, and fertilization on yield and agronomic traits of waxy sorghum Qiangao 7. Jiangsu Agricultural Sciences, 45, 84-88.

\section{Copyrights}

Copyright for this article is retained by the author(s), with first publication rights granted to the journal.

This is an open-access article distributed under the terms and conditions of the Creative Commons Attribution license (http://creativecommons.org/licenses/by/4.0/). 